Springer $\underline{\text { Springer }}$ Springer

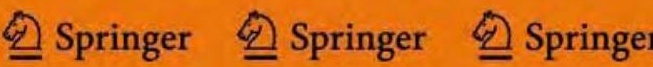

Die positiven Effekte einer Hormonersatztherapie zeigen sich auch in niedriger Dosierung.

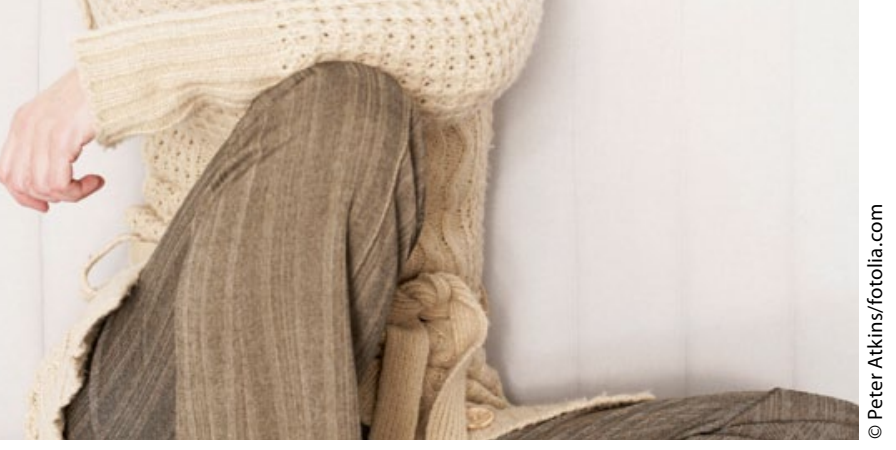

\title{
Hormonersatztherapie: Wie wenig darf es sein?
}

_ Zur Minderung der Risiken soll eine postmenopausale Hormontherapie (HRT) in der niedrigsten wirksamen Dosis verabreicht werden. Wie viel oder wenig ist das? Wie Prof. Inka Wiegratz, Frankfurt, berichtete, waren in den Studien zu vasomotorischen Beschwerden mindestens 0,5 mg orales Estradiol nötig, um eine signifikant bessere Wirkung als mit Placebo zu erzielen. Für konjugierte Östrogene betrug die niedrigste effektive Dosis 0,3 mg. Transdermales Estradiol linderte sogar schon in der ultraniedrigen Dosierung von $14 \mu \mathrm{g}$ Hitzewallungen signifikant. Höhere Dosierungen waren bei allen Östrogenen mit einer höheren Wirksamkeit verbunden. Wiegratz betonte jedoch, dass es sich bei den genannten Dosierungen um Mittelwerte handele: „Es kann sein, dass eine Frau schon mit 0,25 mg oralem Estradiol auskommt. Das Behandlungsziel ist ja auch nicht immer 100\%ige Beschwerdefreiheit." Bei unzureichender Wirksamkeit könne es sich lohnen, erst einmal abzuwarten, so Wiegratz. In den Studien ging die Intensität der Hitzewallungen zwar schon innerhalb von vier Wochen zurück, der maximale Effekt wurde aber erst nach acht bis zwölf Wochen erreicht. Eine Verstärkung der Östrogenwirkung ließ sich durch die Kombination mit Norethisteronacetat erreichen, möglicherweise über die Aromatisierung zu Estradiol.

Ultraniedrige Östrogendosierungen eignen sich auch zur topischen Behandlung einer Vaginalatrophie: Vaginaltabletten mit 10-25 $\mu \mathrm{g}$ Estradiol führen wegen der guten Resorption und Metabolisierung im Scheidenepithel nur zu einem geringfügigen Anstieg der Östradiolspiegel im Plasma; lediglich an Tag 1 der Therapie, bei noch atrophem Vaginalepithel, werden höhere Werte gemessen. Wegen der geringen systemischen Wirksamkeit ist laut Wiegratz keine Proliferation der Uterusschleimhaut zu erwarten und daher auch kein Gestagen erforderlich.

Auch ein weiterer günstiger Effekt der HRT geht selbst mit ultraniedrig dosiertem Östrogen nicht ganz verloren: Sowohl mit 0,25 mg Estradiol oral als auch mit $14 \mu \mathrm{g}$ Estradiol transdermal wurde eine leichte Zunahme der Knochendichte beobachtet.

Erste Studienergebnisse deuten darauf hin, dass sich unerwünschte Wirkungen einer HRT am Brustdrüsengewebe durch niedrig dosierte Präparate vermeiden lassen. Weder mit $14 \mu \mathrm{g}$ Estradiol transdermal noch mit $0,5 \mathrm{mg}$ Estradiol oral erhöhte sich die mammografische Dichte. Dies sei „beruhigend", so Wiegratz. Über die Auswirkungen auf das Brustkrebsrisiko lassen die kurzen Beobachtungszeiten allerdings noch keine Aussage zu.

Dr. Beate Schumacher

Wiegratz I. "Niedrigdosierte Hormontherapie: Ist weniger mehr?"; Hauptsitzung "Menopause", 12.10.2012

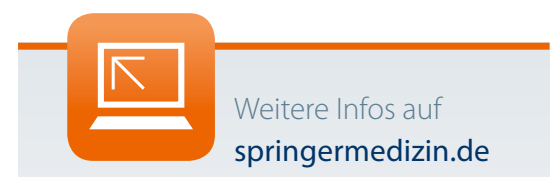

Weitere Beiträge vom 59. DGGG-

Kongress und aktuelle Interviews

finden Sie in unserem Dossier unter

www.springermedizin.

de/3550324
Springer Springer $\underline{\text { Springer }}$

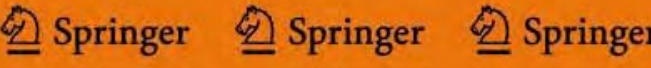

Springer $\underline{\text { Springer }}$ Springer

Springer $\underline{\text { Springer }}$ Springe

Springer Springer $\underline{\text { Springer }}$

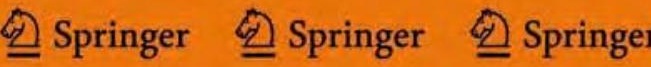

은 Springer $\underline{\text { Springer }}$ Springer

Springer $\underline{\text { Springer }}$ Springe

Springer Springer 2 Springer

Springer Springer Springe

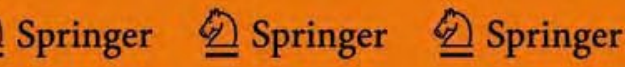

Q Springer Springer Springes

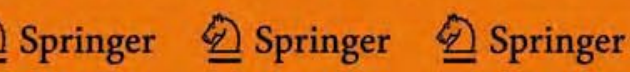

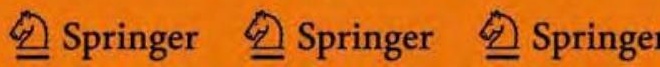

Springer Springer 2 Springer

Springer Springer $\underline{\text { Springe }}$

은 Springer $\underline{\text { Springer }}$ Springer

Springer $\underline{\text { Springer }}$ Springe

Springer Springer $\underline{\text { Springer }}$

Springer Springer Springe

Springer 을 Springer Springer

Q Springer Springer $\underline{\text { Springe }}$

Springer Springer Springer

Q Springer $\underline{\text { Springer }}$ Springer

Springer $\underline{\text { Springer }}$ Springer

Q Springer Springer $\underline{\text { Springes }}$

Springer $\underline{\text { Springer }}$ Springer

Q Springer Springer $\underline{\text { Springe }}$

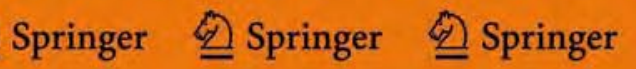

Springer Springer Springe

Springer $\underline{\text { Springer }}$ Springer

은 Springer Springer $\underline{\text { Springe }}$

Springer Springer 